\title{
Single-Incision Mini Sling Compared with Transobturator Sling for Treating Stress Urinary Incontinence in Women
}

Yahia Abd El Salam Wafa, Ahlam El Said Kamel, Mohammed Maghawry Maghawry El Sayed*

Department of Obstetrics and Gynecology, Faculty of Medicine, Al-Azhar University

*Corresponding author: Mohammed Maghawry Maghawry El Sayed, Email: dr_maghwry777@yahoo.com, Mobile: (+20)01224777838

\begin{abstract}
Background: Stress urinary incontinence (SUI) is a common disorder that affects a large number of women and their quality of life. It is defined as the involuntary leakage of urine on effort or exertion, or on sneezing or coughing according to the standardized terminology of the International Continence Society.

Objective: The aim of this study was to compare both the efficacy and safety of the single-incision mini- sling (SIMS) against the traditional trans-obturator (TOT) sling for the surgical management of SUI in women including; objective and subjective cure rates, patient's satisfaction, and complication rates.

Patients and methods: This study was a prospective, double-blind clinical trial conducted on 100 women complaining of symptoms of stress urinary incontinence, with or without urge incontinence, based on patient complaint, cough stress test and ICIQ-SF score.

Results: Regarding the cough stress test after one year follow up, the number of participants who reported negative results showed continuous progress in group (B) [42 women $(89 \%)$ ] after $38(79.2 \%)$ in $6^{\text {th }}$ month visit. On the other hand, group (A) women showed a little drop [44 women $(91.7 \%)$ ] after $46(93.9 \%)$ in $6^{\text {th }}$ month visit. Yet, the two groups showed insignificant difference at this follow up stage $(\mathrm{P}=0.74)$.

Conclusion: The 1-year follow-up results of this prospective trial indicate that both procedures appear to be equally effective for the treatment of SUI as regard the objective cure rates, whereas the SIMS procedure showed higher subjective cure rates than the TOT procedure.
\end{abstract}

Keywords: Single-Incision Mini Sling, Transobturator Sling, Stress Urinary Incontinence, Women, clinical trial.

\section{INTRODUCTION}

Management options vary between conservative and surgical procedures. Conservative approaches for treatment of stress urinary incontinence (SUI) include pelvic floor exercises, electrical stimulation, laser application and duloxetine therapy ${ }^{(\mathbf{1})}$.

Although there is renewed interest in conservative therapies for stress urinary incontinence, surgery remains the primary choice in managing this condition. Surgical options include paravaginal defect repair, the Marshall-Marchetti-Krantz procedure, open and laparoscopic Burch urethropexy, and sling procedures ${ }^{(1)}$. As regards sling use, it was found that midurethral slings techniques achieved high cure rates in women with SUI and have become the mainstay for surgical treatment of SUI in women over the last 2 decades ${ }^{(2)}$. One of the modalities of such procedures is the transobturator midurethral tape (TOT). It was introduced to minimize the complications of the previous retropubic tapes, which include injury to the bladder, major vessels, and bowel. TOT has shown similar safety and efficacy to Tension-free vaginal tape (TVT) in a recently published randomized trials and meta-analyses (3).

In an effort to maintain efficacy while eliminating some of the side effects, a new generation of tapes has been developed, called 'single incision tapes or 'mini-slings'. They are designed to be shorter (in length) than standard mid-urethral slings and do not penetrate the tissues as deeply as standard slings. It was therefore thought that they would cause fewer side effects while being effective ${ }^{(4)}$. Single incision tapes have a number of potential advantages that attracted the attention of many surgeons worldwide: shorter length polypropylene mesh, insertion through a single vaginal incision, avoiding both retropubic and groin trajectories (in retropubic tension-free vaginal tape (RP-TVT) and transobturator tension-free vaginal tapes (TO-TVT)); and the ability to perform the procedure under local anaesthesia and therefore a shorter recovery and earlier return to normal activities ${ }^{(2)}$.

\section{AIM OF THE WORK}

The aim of this study was to compare both the efficacy and safety of the single-incision mini- sling against the traditional trans-obturator sling for the surgical management of SUI in women including; objective and subjective cure rates, patient's satisfaction, and complication rates.

\section{PATIENTS AND METHODS} Study Design:

The present study is a prospective clinical study aimed to compare both the efficacy and the safety of the single-incision mini sling (SIMS) and TOT sling for treating stress urinary incontinence in women.

\section{Study Setting and Timing:}

- Obstetrics and Gynecology Department of AlHussein hospital, Al-Azhar University, Cairo Egypt.

- Obstetrics and Gynecology Department of Ahmad Maher teaching hospital, General Organization of Teaching Hospitals and Institutes, Cairo Egypt. 
Study period: between $1^{\text {st }}$ of March 2016 till $30^{\text {th }}$ of March 2019.

Ethical approval and written informed consent: An approval of the study was obtained from Al- Azhar University Academic and Ethical Committee. Every patient signed an informed written consent for acceptance of the operation.

\section{Measured Outcome:}

The objective cure was defined as a negative cough stress test, and the subjective cure was defined as zero incontinence episodes on the ICIQ-SF. The primary outcome of this study was to test the hypothesis that there was no difference between SIMS and TOT regarding both the objective and the subjective cure rates. The secondary outcome was pain perception (VAS score) and complications incurred at one-year follow up between the two procedures.

\section{Inclusion Criteria:}

Women diagnosed with stress or mixed urinary incontinence based on the patient's main complaint of involuntary leakage of urine upon coughing, sneezing, straining or lifting heavy objects) and/ or urgency were eligible for the study. Stress incontinence had to be the main bothering symptom defined as more episodes of leakage due to coughing or physical exertion than with urgency. Positive cough stress tests with the bladder holding 300 to $400 \mathrm{~mL}$ and fulfilling the International Consultation on Incontinence Questionnaire - Short Form (ICIQ-SF) were used to confirm the diagnosis.

\section{Exclusion criteria:}

Women with history of previous mid urethral sling procedure, presence of a connective tissue disorders, advanced pelvic organ prolapse (Pelvic Organ Prolapse Quantification. stage 3), residual urine volume $>100 \mathrm{~mL}$, previous pelvic radiotherapy, chemotherapy or pregnancy were excluded from the study.

\section{Preoperative Considerations:}

All patients were subjected to the following baseline assessment by history, clinical examinations, investigation as well as answering questionnaires.

\section{$>$ History Taking}

This was carried out to detect the presenting and the dominating symptoms of stress incontinence. The evaluation included age, residency, educational level, menstrual history and obstetric history including obstetrical complications. The past history of diseases which might elevate intra-abdominal pressure as chronic cough and constipation, neurological diseases, medical disorders of significance as diabetes, surgical operations whether abdominal or vaginal and any medications used were considered.

\section{$>$ Clinical Examination:}

All patients were subjected to complete clinical examination including both general and local examinations. Local examination was performed by the researcher himself and a senior consultant who was the chief of the surgical team. In the standing position, the cough stress test was performed at a bladder volume of $300 \mathrm{~mL}$. The results of the cough stress test were considered positive if urine leakage occurred with a cough or negative if no urine leakage was seen and the bladder volume was recorded ${ }^{(5)}$.

\section{$>$ Preoperative Laboratory Investigations:}

Blood samples were drawn from each patient in the study as a routine preoperative protocol. Blood tests were; complete blood count (CBC), liver function tests, renal function tests, fasting and 2 hours- postprandial blood sugar and coagulation profile. Moreover, a midstream urine samples were collected for analysis and culture if needed. Also, an ECG was requested for each patient older than 35 years old for anesthetic purposes.

\section{$>$ Randomization}

On the day of surgery, all women were randomized into two groups; the first group were subjected to the single-incision mini-sling; (SIMS) Altis sling (Coloplast Corp, Minneapolis Minnesota, USA) and the second had the standard trans-obturator tape; (TOT) (Obtryx II Transobturator Sling System - HaloBoston Scientific, Natick, MA, USA) using a computergenerated block randomization sequence, with allocation to each group being performed via a series of opaque envelopes.

\section{$>$ Surgical Procedures:}

All surgeries were performed by a single surgeon (A.E.). Prior to surgery, parenteral antibiotics were injected (1.5 gm Ampicillin/ Sulbactam or $80 \mathrm{mg}$ Gentamicin). Following induction of anesthesia (whether general or spinal) the patients were carefully placed in a slightly exaggerated dorsal lithotomy position with appropriate pressure points adequately padded. The operative field was cleaned with a standard antiseptic agent and draped, with care being taken to keep the groin folds in the operative field. A 14-Gauge urethral catheter was inserted into the bladder and the bladder was emptied.

The surgical procedure was performed either alone or in combination with other surgical procedures e.g. anterior colporrhaphy, posterior colpoperineorrhaphy or perineal repair. Diagnostic cystoscopy was not routinely performed.

Duration of anti-incontinence surgery was recorded dependently from other concomitant procedure. Any complications were recorded and managed accordingly.

\section{- Surgical technique of SIMS:}

The Altis sling is made of a $0.8 \mathrm{~mm}$ diameter monofilament polypropylene mesh, knitted into a sling $1.1 \mathrm{~cm}$ wide by $7.75 \mathrm{~cm}$ long. One end of the sling assembly connects to a short length of USP size I monofilament polypropylene suture and to a polypropylene static (non-tensioning) anchor. Whereas, the second side of the sling assembly terminates to a longer section of USP size I monofilament 
polypropylene suture. This suture is then positioned through the second anchor that is dynamic with an integrated tensioning system. The tensioning capability is accomplished by threading the suture through a polyurethane tensioning collar which is assembled onto the anchor. The anchor/suture assembly wraps around the anchor, resulting in a gap between the anchor and the collar providing constant pressure on the suture, preventing it from moving on its own, or during a pelvic stress event. Tensioning is achieved by pulling on the suture loop, thus applying increased tension of the sling to the urethra until desired support is achieved. The Altis anchors perform an acute mechanical role of maintaining the desired position and tension through the acute postoperative tissue healing and in-growth phase (Adopted from Altis single-incision sling system product brochure, Coloplast).

\section{- Surgical technique of TVT-O}

The Obtryx Sling System - Halo is a sterile, single use system consisting of a polypropylene knitted mesh protected by a disposable plastic sleeve. At the distal ends of the mesh assembly there are association loops designed to be placed in the needle slot of the distal end of the delivery device. The disposable delivery device consists of a handle with a stainless steel needle. The needle is designed to facilitate the passage of the mesh assembly through bodily tissues for placement through the obturator foramen (Adopted from Obtryx ${ }^{\mathrm{TM}}$ -Transobturator Mid-Urethral Sling System, Product Review for the Purchasing Committee).

\section{$>$ Post Operative Care and Follow-Up:}

Post operatively, vital signs, vaginal bleeding or hematomas were monitored and recorded. Pain was assessed by Visual Analogue Scale (VAS) which is a continuous scale comprised of a horizontal (HVAS) or vertical (VVAS) line, 10 centimeters $(100 \mathrm{~mm})$ in length, anchored by 2 verbal descriptors, one for each symptom extreme. Hospital discharge occurred 24-h after surgery after three spontaneous micturitions with post voiding residual (PVR) $<100 \mathrm{ml}$ evaluated by ultrasound and/ or catheterization.

Complications were managed accordingly. Intraoperative bladder injury was managed by urgent cystoscopy and repair of injured site by Vicryl 2/0 sutures if needed. Transurethral bladder drainage by Foley catheter was maintained for 7 days postoperatively. In case of hematoma, it was evacuated and the feeding vessel was secured. The procedure was postponed for another sitting and the case was excluded from the study. Follow-up visits were scheduled by a physician not involved with the surgeries. Follow-up were at day 7 , and then $1,3,6$, and 12 months after the procedure. On the $7^{\text {th }}$ day visit, a brief history of the patient pain profile, fever, inability to void easily, frequency, urgency, dysuria, leakage of urine with cough and appearance of vaginal discharge were discussed and recorded. Examination of signs of inflammation or infection with cough stress test and post voiding residual were assessed and recorded.

The $1^{\text {st }}, 3^{\text {rd }}, 6^{\text {th }}$ and $12^{\text {th }}$ - month follow up visits aimed to ask the patients about the modifications in voiding habits regarding number of micturitions, incontinence episodes during the day, pain score (VAS) and if they faced voiding difficulties coinciding with fulfilling of (ICIQ-SF). Moreover, a cough stress test was performed as an objective measure for surgical success. All the data was collected in a specially designed sheet for each patient.

\section{Statistical Analysis}

For quantitative data, the Shapiro-Wilk test for normality was performed. For normally distributed data, values were expressed as mean \pm standard deviation and Independent Samples $\mathrm{T}$ test was performed for comparison between two groups. For data that were not normally distributed median and interquartile range (expressed as $25^{\text {th }}-75^{\text {th }}$ percentiles) were calculated and Mann-Whitney test was used for comparison between the two groups and Friedman's two-way analysis of variance for repeated measurements. As regards qualitative data, they were represented as frequency (number and percentage) and either Pearson's Chi square test for independence, Fisher's exact test or FisherFreeman-Halton exact test was used to examine association between two variables. Significance was adopted at $\mathrm{p}<0.05$ for interpretation of results of tests.

\section{The following tests were done:}

- Independent-samples t-test of significance was used when comparing between two means.

- Chi-square $\left(\mathrm{x}^{2}\right)$ test of significance was used in order to compare proportions between two qualitative parameters.

- The confidence interval was set to $95 \%$ and the margin of error accepted was set to $5 \%$. The p-value was considered significant as the following:

- Probability (P-value)

- P-value $\leq 0.05$ was considered significant.

- P-value <0.001 was considered as highly significant.

- P-value >0.05 was considered insignificant.

\section{RESULTS}

In total, 100 women fulfilled the inclusion criteria. They were randomly divided in two groups whereas group (A) was subjected to SIMS insertion, while the second group (B) was subjected to TOT for the treatment of stress urinary incontinence. During the study, some patients missed the follow up visits; consequently, they were excluded from the study if two consecutive visits were missed as shown in figure (1). 


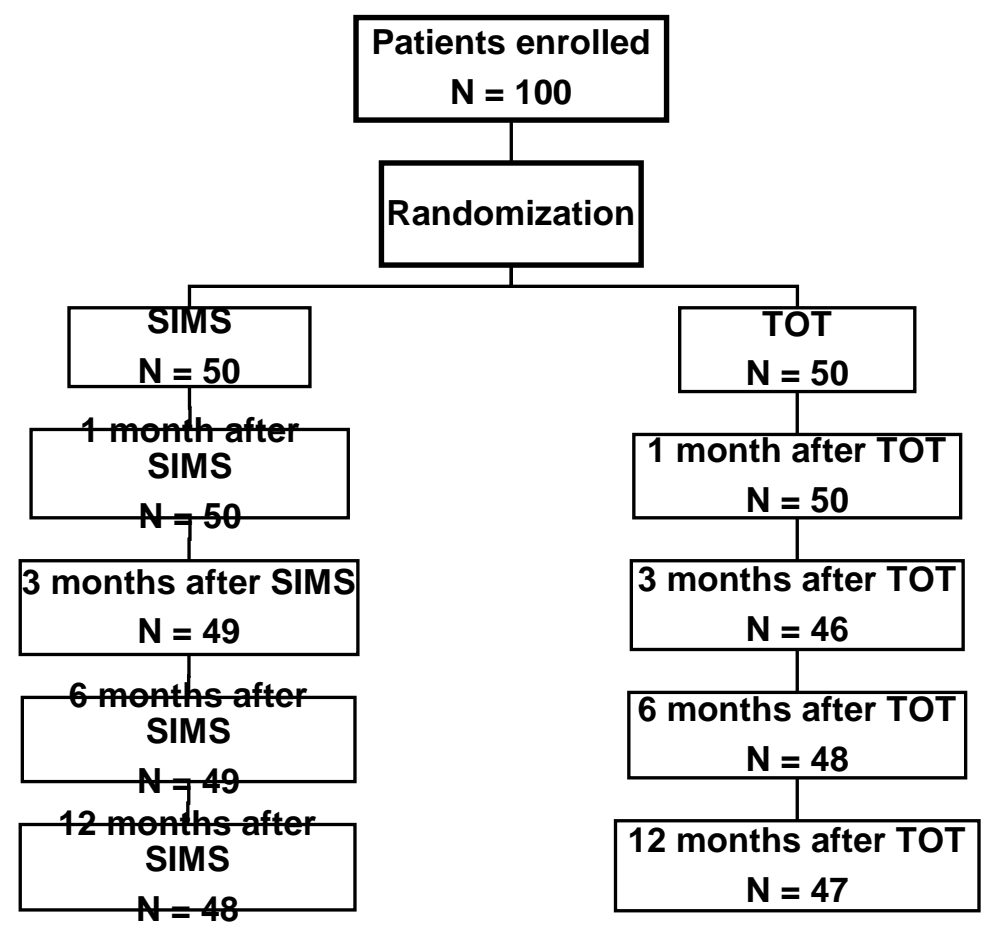

Figure (1): Flow-chart of study population. SIMS, single-incision mid-urethral sling; TOT, transobturator tape.

- Socio-demographic data of the studied groups:

The socio-demographic data of the studied groups regarding age, residence, occupation and education level showed insignificant difference between both groups.

$>$ Age:

The age of women included in this study ranged between 27 and 65 years with a mean of $46.3 \pm 10.5$ years. This mean was 44.3 years in group (A) (SIMS procedure) while 48.3 years in group (B) (TOT procedure) without any statistically significance $(\mathrm{P}=$ 0.057) as shown in table (1).

\section{$>$ Residency:}

There was nearly equal distribution of women regarding their residency either from urban areas 56\% or rural areas $44 \%(\mathrm{p}=0.17)$ as shown in table (1).

Occupation:

Table (1): Descriptive data of the 100 women participating in the two study groups.

\begin{tabular}{|c|c|c|c|c|c|c|c|c|c|}
\hline \multirow{2}{*}{\multicolumn{2}{|c|}{ Variable }} & \multicolumn{6}{|c|}{ Groups } & \multicolumn{2}{|c|}{ Tests of significance } \\
\hline & & \multirow{2}{*}{\multicolumn{2}{|c|}{$\begin{array}{c}\begin{array}{c}\text { TOT } \\
(\mathbf{n}=\mathbf{5 0})\end{array} \\
30.0-65.0\end{array}$}} & \multicolumn{2}{|c|}{$\begin{array}{c}\text { SIMS } \\
(\mathbf{n}=\mathbf{5 0})\end{array}$} & \multicolumn{2}{|c|}{$\begin{array}{c}\text { Total } \\
(\mathbf{n}=\mathbf{1 0 0})\end{array}$} & Test statistic & p \\
\hline \multirow{2}{*}{ Age } & Min- Max & & & \multirow{2}{*}{\multicolumn{2}{|c|}{$\frac{257.0-65.0}{44.3 \pm 9.8}$}} & \multirow{2}{*}{\multicolumn{2}{|c|}{$\frac{27.0-65.0}{46.3 \pm 10.5}$}} & \multirow[t]{2}{*}{$\mathrm{t}=1.929$} & \multirow[t]{2}{*}{0.057} \\
\hline & Mean \pm SD & \multicolumn{2}{|c|}{$48.3 \pm 10.8$} & & & & & & \\
\hline \multirow{2}{*}{ Residency } & Urban & 32 & $64.0 \%$ & 24 & $48.0 \%$ & 56 & $56.0 \%$ & \multirow[t]{2}{*}{$\mathrm{X}^{2} \mathrm{ChS}=2.597$} & \multirow[t]{2}{*}{0.107} \\
\hline & Rural & 18 & $36.0 \%$ & 26 & $52.0 \%$ & 44 & $44.0 \%$ & & \\
\hline \multirow{2}{*}{ Occupation } & Working & 3 & $6.0 \%$ & 7 & $14.0 \%$ & 10 & $10.0 \%$ & \multirow[t]{2}{*}{$\mathrm{X}_{\mathrm{ChS}}^{2}=1.778$} & \multirow[t]{2}{*}{0.182} \\
\hline & Not working & 47 & $94.0 \%$ & 43 & $86.0 \%$ & 90 & $90.0 \%$ & & \\
\hline \multirow{3}{*}{$\begin{array}{c}\text { Educational } \\
\text { level }\end{array}$} & Illiterate & 12 & $24.0 \%$ & 7 & $14.0 \%$ & 19 & $19.0 \%$ & \multirow[t]{3}{*}{$\mathrm{X}_{\mathrm{ChS}}^{2}=1.949$} & \multirow[t]{3}{*}{0.377} \\
\hline & Mid-level & 27 & $54.0 \%$ & 28 & $56.0 \%$ & 55 & $55.0 \%$ & & \\
\hline & High level & 11 & $22.0 \%$ & 15 & $30.0 \%$ & 26 & $26.0 \%$ & & \\
\hline \multirow{2}{*}{ Special habits } & None & 47 & $94.0 \%$ & 46 & $92.0 \%$ & 93 & $93.0 \%$ & \multirow[t]{2}{*}{$\mathrm{X}^{2} \mathrm{ChS}=0.145$} & \multirow[t]{2}{*}{0.695} \\
\hline & Smoker & 3 & $6.0 \%$ & 4 & $8.0 \%$ & 7 & $7.0 \%$ & & \\
\hline
\end{tabular}

The great majority of women on both groups had no jobs $(90 \%)$. Despite ranging from $86 \%$ in group (A) to $94 \%$ in group (B), however, this was statistically insignificant as shown in table (1).

\section{$>$ Educational level:}

According to educational level, the highest percentage of the studied groups was in women with mid- level of education in both group A and B (56.0 $\%$ \& $54.0 \%$ respectively) followed by those with high education (26\% in both groups). While, the lowest percentage of educational level was about $19 \%$ in both groups as shown in table (1).

\section{$>$ Special habits:}

Most of the studied women were nonsmokers; representing about $92 \%$ in group (A) and $94 \%$ in group (B). Otherwise, other special habits were irrelevant. 


\section{- Post-operative follow- up}

The duration of hospital stay was almost 24 hours after surgery in most of cases $(88 \%$ in group (A) and $84 \%$ in group (B)). Only 6 women in group (A) $(12 \%)$ and 8 women in group (B) $(16 \%)$ needed more than 24 hours $(\mathrm{P}=0.564)$ as shown in table (2).

Regarding post-operative groin pain (first day), it ranged from 3 to 7 on the visual analogue scale (VAS) with a mean of $4.7 \pm 1.2$ in group (A) and from 4 to 7 with a mean of $5.5 \pm 1.1$ in group (B) which represented a statistically significant difference
( $\mathbf{P}=\mathbf{0 . 0 0 1 )}$ as shown in table (2).There was no statistically significant difference between both groups regarding; cough stress test, post-voiding residual urine and urine retention $(\mathrm{P}=0.207,0.893$ and 0.692 , respectively) as shown in table (2).

Eleven women from group (A) and nine from group (B) complained early postoperative partial urinary outlet obstruction, leading to increased residual bladder volume $(>100 \mathrm{~mL})$. This was treated using intermittent bladder catheterization for up to 1 week.

Table (2): Comparison between both studied groups as regard post-operative complications and hospital stay.

\begin{tabular}{|c|c|c|c|c|c|c|c|c|c|}
\hline & \multicolumn{6}{|c|}{ Groups } & \multicolumn{2}{|c|}{ Tests of significance } \\
\hline & & \multicolumn{2}{|c|}{$\begin{array}{c}\text { TOT } \\
(\mathbf{n}=\mathbf{5 0})\end{array}$} & \multicolumn{2}{|c|}{$\begin{array}{c}\text { SIMS } \\
(\mathbf{n}=\mathbf{5 0})\end{array}$} & \multicolumn{2}{|c|}{$\begin{array}{c}\text { Total } \\
(\mathbf{n}=\mathbf{1 0 0})\end{array}$} & Test statistic & $\mathbf{p}$ \\
\hline \multirow[t]{2}{*}{ Pain 1 (VAS) } & Min- Max & \multicolumn{2}{|c|}{$4.0-7.0$} & \multicolumn{2}{|c|}{$3.0-7.0$} & \multicolumn{2}{|c|}{$3.0-7.0$} & \multirow[t]{2}{*}{$t=3.309$} & \multirow[t]{2}{*}{$0.001^{*}$} \\
\hline & Mean \pm SD & \multicolumn{2}{|c|}{$5.5 \pm 1.1$} & \multicolumn{2}{|c|}{$4.7 \pm 1.2$} & \multicolumn{2}{|c|}{$5.1 \pm 1.2$} & & \\
\hline \multirow[t]{3}{*}{ Cough stress test 1} & Positive & 23 & $46.0 \%$ & 17 & $34.0 \%$ & 40 & $40.0 \%$ & \multirow[t]{3}{*}{$\mathrm{X}^{2} \mathrm{ChS}=2.088$} & \multirow[t]{3}{*}{0.207} \\
\hline & Negative & 22 & $44.0 \%$ & 30 & $60.0 \%$ & 52 & $52.0 \%$ & & \\
\hline & Cath & 5 & $10.0 \%$ & 3 & $6.0 \%$ & 8 & $8.0 \%$ & & \\
\hline \multirow{3}{*}{$\begin{array}{l}\text { Post Voiding residual } \\
1\end{array}$} & Less 100 & 35 & $70.0 \%$ & 36 & $72.0 \%$ & 71 & $71.0 \%$ & \multirow[t]{3}{*}{$\mathrm{X}^{2} \mathrm{ChS}=0.018$} & \multirow[t]{3}{*}{0.893} \\
\hline & More 100 & 10 & $20.0 \%$ & 11 & $22.0 \%$ & 21 & $21.0 \%$ & & \\
\hline & Catheter & 5 & $10.0 \%$ & 3 & $6.0 \%$ & 8 & $8.0 \%$ & & \\
\hline \multirow[t]{2}{*}{ Hospital Stay } & 24 hrs & 42 & $84.0 \%$ & 44 & $88.0 \%$ & 86 & $86.0 \%$ & \multirow[t]{2}{*}{$\mathrm{X}^{2} \mathrm{ChS}=0.332$} & \multirow[t]{2}{*}{0.564} \\
\hline & $>24 \mathrm{hrs}$ & 8 & $16.0 \%$ & 6 & $12.0 \%$ & 14 & $14.0 \%$ & & \\
\hline \multirow[t]{3}{*}{ Urine retention 1} & Positive & 9 & $18.0 \%$ & 11 & $22.0 \%$ & 20 & $20.0 \%$ & \multirow[t]{3}{*}{$\mathrm{X}_{\mathrm{ChS}}^{2}=0.157$} & \multirow[t]{3}{*}{0.692} \\
\hline & Negative & 36 & $72.0 \%$ & 36 & $72.0 \%$ & 72 & $72.0 \%$ & & \\
\hline & Catheter & 5 & $10.0 \%$ & 3 & $6.0 \%$ & 8 & $8.0 \%$ & & \\
\hline
\end{tabular}

One week post-operatively, the statistically significant difference in the level of the groin pain (VAS) was still existed between the two groups (despite much improvement) with a mean of $2.2 \pm 1.5$ for group (A) and $3.6 \pm 1.7$ for group (B) $(\mathbf{P}=\mathbf{< 0 . 0 0 1})$. Additionally, the presence of groin pain itself showed extreme variability between the two groups (6\% in group (A) compared to $70 \%$ in group (B)) $(\mathbf{P}=<\mathbf{0 . 0 0 1})$ as shown in table (3).Moreover, there was no statistically significant difference between both groups regarding; cough stress test, post-voiding residual urine, excessive vaginal discharge, subjective emptying problems, urge incontinence and wound infection $(\mathrm{P}>0.05)$ as shown in table (3).

Variable degrees of urge incontinence were presented among women in both groups. Six women in group (A) and four women in group (B) were prescribed medications due to severe urge incontinence.

Table (3): Comparison between both studied groups as regard one-week post-operative pain score and complications

\begin{tabular}{|c|c|c|c|c|c|c|c|c|c|}
\hline & \multicolumn{6}{|c|}{ Groups } & \multicolumn{2}{|c|}{ Tests of significance } \\
\hline & & \multirow{2}{*}{\multicolumn{2}{|c|}{$\begin{array}{c}\begin{array}{c}\text { TOT } \\
(\mathbf{n}=\mathbf{5 0})\end{array} \\
0.0-6.0\end{array}$}} & \multicolumn{2}{|c|}{$\begin{array}{c}\text { SIMS } \\
(\mathbf{n}=\mathbf{5 0})\end{array}$} & \multicolumn{2}{|c|}{$\begin{array}{c}\text { Total } \\
(\mathbf{n}=\mathbf{1 0 0})\end{array}$} & Test statistic & $\mathbf{p}$ \\
\hline \multirow[t]{2}{*}{ Pain 2 (VAS) } & Min- Max & & & \multirow{2}{*}{\multicolumn{2}{|c|}{$\frac{0.0-5.0}{2.2 \pm 1.5}$}} & \multirow{2}{*}{\multicolumn{2}{|c|}{$\begin{array}{c}0.0-6.0 \\
29+17\end{array}$}} & \multirow[t]{2}{*}{$\mathrm{t}=4.330$} & \multirow[t]{2}{*}{$<0.001 *$} \\
\hline & Mean \pm SD & \multicolumn{2}{|c|}{$3.6 \pm 1.7$} & & & & & & \\
\hline \multirow[t]{2}{*}{ Cough stress test 2} & Positive & 23 & $46.0 \%$ & 20 & $40.0 \%$ & 43 & $43.0 \%$ & \multirow[t]{2}{*}{$\mathrm{X}_{\mathrm{ChS}}^{2}=0.367$} & \multirow[t]{2}{*}{0.545} \\
\hline & Negative & 27 & $54.0 \%$ & 30 & $60.0 \%$ & 57 & $57.0 \%$ & & \\
\hline \multirow[t]{3}{*}{ Post Voiding residual 2} & Less 100 & 40 & $80.0 \%$ & 42 & $84.0 \%$ & 82 & $82.0 \%$ & \multirow[t]{3}{*}{$\mathrm{X}^{2} \mathrm{ChS}=0.271$} & \multirow[t]{3}{*}{0.603} \\
\hline & More 100 & 10 & $20.0 \%$ & 8 & $16.0 \%$ & 18 & $18.0 \%$ & & \\
\hline & Absent & 32 & $64.0 \%$ & 45 & $90.0 \%$ & 77 & $77.0 \%$ & & \\
\hline \multirow[t]{2}{*}{ Vaginal discharge } & Excessive & 16 & $32.0 \%$ & 20 & $40.0 \%$ & 36 & $36.0 \%$ & \multirow[t]{2}{*}{$\mathrm{X}_{\mathrm{ChS}}^{2}=0.694$} & \multirow[t]{2}{*}{0.405} \\
\hline & Usual & 34 & $68.0 \%$ & 30 & $60.0 \%$ & 64 & $64.0 \%$ & & \\
\hline \multirow{2}{*}{$\begin{array}{l}\text { Subjective emptying } \\
\text { problems } 2\end{array}$} & Present & 7 & $14.0 \%$ & 9 & $18.0 \%$ & 16 & $16.0 \%$ & \multirow[t]{2}{*}{$\mathrm{X}^{2} \mathrm{ChS}=0.298$} & \multirow[t]{2}{*}{0.585} \\
\hline & Absent & 43 & $86.0 \%$ & 41 & $82.0 \%$ & 84 & $84.0 \%$ & & \\
\hline \multirow[t]{2}{*}{ Urge incontinence 2} & Present & 21 & $42.0 \%$ & 18 & $36.0 \%$ & 39 & $39.0 \%$ & \multirow[t]{2}{*}{$\mathrm{X}_{\mathrm{ChS}}^{2}=0.378$} & \multirow[t]{2}{*}{0.539} \\
\hline & Absent & 29 & $58.0 \%$ & 32 & $64.0 \%$ & 61 & $61.0 \%$ & & \\
\hline \multirow[t]{2}{*}{ Groin Pain 2} & Present & 35 & $70.0 \%$ & 3 & $6.0 \%$ & 38 & $38.0 \%$ & \multirow[t]{2}{*}{$\mathrm{X}_{\mathrm{ChS}}^{2}=43.463$} & \multirow[t]{2}{*}{$<0.001 *$} \\
\hline & Absent & 15 & $30.0 \%$ & 47 & $94.0 \%$ & 62 & $62.0 \%$ & & \\
\hline \multirow[t]{2}{*}{ Wound infection 2} & Present & 6 & $12.0 \%$ & 5 & $10.0 \%$ & 11 & $11.0 \%$ & \multirow[t]{2}{*}{$\mathrm{X}_{\mathrm{ChS}}^{2}=0.102$} & \multirow[t]{2}{*}{0.749} \\
\hline & Absent & 44 & $88.0 \%$ & 45 & $90.0 \%$ & 89 & $89.0 \%$ & & \\
\hline
\end{tabular}


- Follow up visits

Follow up was performed at $1^{\text {st }}, 3^{\text {rd }}, 6^{\text {th }}$ and $12^{\text {th }}$ month. At these recalls, the participants were asked to fill the ICIQ-SF about persistent urinary stress incontinence, amount of leaked urine, interference with everyday life, difficulties in micturition i.e. voiding dysfunction, urgency and other $\mathrm{OAB}$ symptoms and changes occurred in voiding habits.

\section{$>$ The first month follow-up:}

Both the cough stress test (objective cure) and the ICIQ-SF score (subjective cure) were statistically insignificant for both groups in the first month $(\mathrm{P}=0.205$ and 0.110$)$ as shown in table (4).

Table (4): Comparison between both studied groups as regard post-operative urinary symptoms and pain scores at the $1^{\text {st }}$ month follow up.

\begin{tabular}{|c|c|c|c|c|c|c|c|c|c|}
\hline & \multicolumn{6}{|c|}{ Groups } & \multicolumn{2}{|c|}{ Tests of significance } \\
\hline & & \multicolumn{2}{|c|}{$\begin{array}{c}\text { TOT } \\
(\mathbf{n}=\mathbf{5 0})\end{array}$} & \multicolumn{2}{|c|}{$\begin{array}{c}\text { SIMS } \\
(\mathrm{n}=\mathbf{5 0})\end{array}$} & \multicolumn{2}{|c|}{$\begin{array}{c}\text { Total } \\
(\mathbf{n}=\mathbf{1 0 0})\end{array}$} & $\begin{array}{c}\text { Test } \\
\text { statistic }\end{array}$ & $\mathbf{P}$ \\
\hline \multirow{2}{*}{$\begin{array}{l}\text { Cough stress test } \\
\text { 1m (objective } \\
\text { cure) }\end{array}$} & Positive & 20 & $40.0 \%$ & 14 & $28.0 \%$ & 34 & $34.0 \%$ & \multirow{2}{*}{$\begin{array}{c}\mathrm{X}^{2} \mathrm{ChS}=1.60 \\
4\end{array}$} & \multirow[t]{2}{*}{0.205} \\
\hline & Negative & 30 & $60.0 \%$ & 36 & $72.0 \%$ & 66 & $66.0 \%$ & & \\
\hline \multirow[t]{3}{*}{ ICIQ-SF score $1 \mathrm{~m}$} & Min-Max & \multicolumn{2}{|c|}{$0.0-21.0$} & \multicolumn{2}{|c|}{$0.0-18.0$} & \multicolumn{2}{|c|}{$0.0-21.0$} & \multirow[t]{3}{*}{$Z=-1.600$} & \multirow[t]{3}{*}{0.110} \\
\hline & $\begin{array}{l}\text { Median } \\
\text { (IQR) }\end{array}$ & \multicolumn{2}{|c|}{$9.0(0.0-11.0)$} & \multicolumn{2}{|c|}{$5.0(0.0-10.0)$} & \multicolumn{2}{|c|}{$8.0(0.0-10.5)$} & & \\
\hline & $\begin{array}{l}\text { Mean } \\
\text { rank }\end{array}$ & \multicolumn{2}{|c|}{55.0} & \multicolumn{2}{|c|}{46.0} & & & & \\
\hline \multirow{6}{*}{$\begin{array}{l}\text { ICIQ question 3-- } \\
\text { 1m }\end{array}$} & Never & 16 & $32.0 \%$ & 20 & $40.0 \%$ & 36 & $36.0 \%$ & \multirow{6}{*}{$\begin{array}{c}\mathrm{X}_{\mathrm{FFH}}^{2}=8.58 \\
7\end{array}$} & \multirow[t]{6}{*}{0.106} \\
\hline & $\begin{array}{l}\text { once/wee } \\
\text { k }\end{array}$ & 5 & $10.0 \%$ & 6 & $12.0 \%$ & 11 & $11.0 \%$ & & \\
\hline & $\begin{array}{l}\mathbf{2 - 3} \\
\text { times/wee } \\
\mathbf{k}\end{array}$ & 16 & $32.0 \%$ & 19 & $38.0 \%$ & 35 & $35.0 \%$ & & \\
\hline & Daily & 5 & $10.0 \%$ & 5 & $10.0 \%$ & 10 & $10.0 \%$ & & \\
\hline & Several & 2 & $4.0 \%$ & 0 & $0.0 \%$ & 2 & $2.0 \%$ & & \\
\hline & All time & 6 & $12.0 \%$ & 0 & $0.0 \%$ & 6 & $6.0 \%$ & & \\
\hline \multirow{2}{*}{$\begin{array}{l}\text { Number of } \\
\text { Micturitions per } \\
\text { day } 1 \mathrm{~m}\end{array}$} & $\begin{array}{l}\text { Min- } \\
\text { Max }\end{array}$ & \multicolumn{2}{|c|}{$5.0-8.0$} & \multicolumn{2}{|c|}{$5.0-8.0$} & \multicolumn{2}{|c|}{$5.0-8.0$} & \multirow[t]{2}{*}{$\mathrm{t}=2.540$} & \multirow[t]{2}{*}{$0.013 *$} \\
\hline & $\begin{array}{l}\text { Mean } \pm \\
\text { SD }\end{array}$ & \multicolumn{2}{|c|}{$6.8 \pm 0.9$} & \multicolumn{2}{|c|}{$6.4 \pm 0.9$} & \multicolumn{2}{|c|}{$6.6 \pm 0.9$} & & \\
\hline \multirow{3}{*}{$\begin{array}{l}\text { Incontinence } \\
\text { episodes per day } \\
\text { 1m }\end{array}$} & Min-Max & & $0-6.0$ & & -5.0 & & -6.0 & $Z=-1.751$ & 0.080 \\
\hline & $\begin{array}{l}\text { Median } \\
\text { (IQR) }\end{array}$ & & $(0.0-4.0)$ & 1.0 & $0.0-2.0)$ & 1.0 & $0.0-2.5)$ & & \\
\hline & $\begin{array}{l}\text { Mean } \\
\text { rank }\end{array}$ & & 55.4 & & 45.6 & & & & \\
\hline Groin Pain 1m & Present & 27 & $54.0 \%$ & 0 & $0.0 \%$ & 27 & $27.0 \%$ & $\mathrm{X}_{\mathrm{ChS}}^{2}=36.9$ & $<0.001 *$ \\
\hline & Absent & 23 & $46.0 \%$ & 50 & $100.0 \%$ & 73 & $73.0 \%$ & 86 & \\
\hline $\begin{array}{l}\text { Post Voiding } \\
\text { residual 1m }\end{array}$ & Less 100 & 46 & $92.0 \%$ & 47 & $\begin{array}{c}94.0 \\
\%\end{array}$ & 93 & $93.0 \%$ & FE & 1.000 \\
\hline & More 100 & 4 & $8.0 \%$ & 3 & $6.0 \%$ & 7 & $7.0 \%$ & & \\
\hline Subjective & Present & 0 & $0.0 \%$ & 0 & $0.0 \%$ & 0 & $0.0 \%$ & FE & 1.000 \\
\hline $\begin{array}{l}\text { emptying } \\
\text { problems 1m }\end{array}$ & Absent & 0 & $0.0 \%$ & 0 & $0.0 \%$ & 0 & $0.0 \%$ & & \\
\hline
\end{tabular}


The third month follow-up:

Table (5): Comparison between both studied groups as regard post-operative urinary symptoms and pain scores at the $3^{\text {rd }}$ month follow up.

\begin{tabular}{|c|c|c|c|c|c|c|c|c|c|}
\hline & \multicolumn{6}{|c|}{ Groups } & \multicolumn{2}{|c|}{ Tests of significance } \\
\hline & & \multicolumn{2}{|c|}{$\begin{array}{c}\text { TOT } \\
(n=46)\end{array}$} & \multicolumn{2}{|c|}{$\begin{array}{c}\text { SIMS } \\
(n=49)\end{array}$} & \multicolumn{2}{|c|}{$\begin{array}{c}\text { Total } \\
(\mathbf{n}=95)\end{array}$} & $\begin{array}{c}\text { Test } \\
\text { statistic }\end{array}$ & $\mathbf{p}$ \\
\hline \multirow[t]{2}{*}{$\begin{array}{l}\text { Cough stress test } 3 \\
\text { (objective cure) }\end{array}$} & Positive & 15 & $\begin{array}{l}32.6 \\
\%\end{array}$ & 5 & $10.2 \%$ & 20 & $21.1 \%$ & \multirow[t]{2}{*}{$\mathrm{X}_{\mathrm{ChS}}^{2}=7.166$} & \multirow[t]{2}{*}{$0.007 *$} \\
\hline & Negative & 31 & $\begin{array}{c}67.4 \\
\%\end{array}$ & 44 & $89.8 \%$ & 75 & $78.9 \%$ & & \\
\hline \multirow[t]{3}{*}{ ICIQ-SF score 3} & Min-Max & \multirow{2}{*}{\multicolumn{2}{|c|}{$\begin{array}{c}0.0-21.0 \\
4.0(0.0- \\
10.0)\end{array}$}} & \multicolumn{2}{|c|}{$0.0-18.0$} & \multicolumn{2}{|c|}{$0.0-21.0$} & \multirow[t]{3}{*}{$\mathrm{Z}=1.298$} & \multirow[t]{3}{*}{0.194} \\
\hline & $\begin{array}{l}\text { Median } \\
\text { (IQR) }\end{array}$ & & & 0.0 & $(0.0-8.0)$ & 4.0 & $0.0-8.0)$ & & \\
\hline & $\begin{array}{l}\text { Mean } \\
\text { rank }\end{array}$ & \multicolumn{2}{|c|}{51.6} & \multicolumn{2}{|c|}{44.6} & & & & \\
\hline \multirow[t]{6}{*}{ ICIQ question 3-3 } & Never & 16 & $\begin{array}{c}34.8 \\
\%\end{array}$ & 25 & $51.0 \%$ & 41 & $43.2 \%$ & \multirow[t]{6}{*}{$\begin{array}{c}\mathrm{X}_{\mathrm{FFH}}^{2}=17.09 \\
8\end{array}$} & \multirow[t]{6}{*}{$0.003 *$} \\
\hline & once/week & 14 & $\begin{array}{c}30.4 \\
\%\end{array}$ & 6 & $12.2 \%$ & 20 & $21.1 \%$ & & \\
\hline & $\begin{array}{l}2-3 \\
\text { times/wee } \\
\text { k }\end{array}$ & 6 & $\begin{array}{c}13.0 \\
\%\end{array}$ & 12 & $24.5 \%$ & 18 & $18.9 \%$ & & \\
\hline & Daily & 2 & $4.3 \%$ & 6 & $12.2 \%$ & 8 & $8.4 \%$ & & \\
\hline & Several & 2 & $4.3 \%$ & 0 & $0.0 \%$ & 2 & $2.1 \%$ & & \\
\hline & All time & 6 & $\begin{array}{c}13.0 \\
\%\end{array}$ & 0 & $0.0 \%$ & 6 & $6.3 \%$ & & \\
\hline \multirow[t]{2}{*}{$\begin{array}{l}\text { Number of } \\
\text { micturitions per day } 3\end{array}$} & $\begin{array}{l}\text { Min- } \\
\text { Max }\end{array}$ & \multicolumn{2}{|c|}{$5.0-8.0$} & \multicolumn{2}{|c|}{$6.0-9.0$} & \multicolumn{2}{|c|}{$5.0-9.0$} & \multirow[t]{2}{*}{$\mathrm{t}=3.590$} & \multirow[t]{2}{*}{$0.001 *$} \\
\hline & $\begin{array}{l}\text { Mean } \pm \\
\text { SD }\end{array}$ & \multicolumn{2}{|c|}{$6.7 \pm 0.9$} & \multicolumn{2}{|c|}{$7.4 \pm 0.9$} & \multicolumn{2}{|c|}{$7.1 \pm 1.0$} & & \\
\hline \multirow{3}{*}{$\begin{array}{l}\text { Incontinence episodes } \\
\text { per day } 3\end{array}$} & Min-Max & \multicolumn{2}{|c|}{$0.0-6.0$} & \multicolumn{2}{|c|}{$0.0-4.0$} & \multicolumn{2}{|c|}{$0.0-6.0$} & $\mathrm{Z}=1.239$ & 0.215 \\
\hline & $\begin{array}{l}\text { Median } \\
\text { (IQR) }\end{array}$ & & $\begin{array}{l}(0.0- \\
.0)\end{array}$ & 1.0 & $(0.0-1.0)$ & 1.0 & $0.0-1.0)$ & & \\
\hline & $\begin{array}{l}\text { Mean } \\
\text { rank }\end{array}$ & & 1.4 & & 44.8 & & & & \\
\hline Groin Pain 3 & Present & 21 & $45.7 \%$ & 0 & $0.0 \%$ & 21 & $22.1 \%$ & $\mathrm{X}_{\mathrm{ChS}}^{2}=28.71$ & $<0.001^{*}$ \\
\hline & Absent & 25 & $54.3 \%$ & 59 & $100.0 \%$ & 74 & $77.9 \%$ & 8 & \\
\hline Post Voiding residual & Less 100 & 46 & $100.0 \%$ & 46 & $93.9 \%$ & 92 & $96.8 \%$ & $\mathrm{FE}$ & 0.243 \\
\hline 3 & More 100 & 0 & $0.0 \%$ & 3 & $6.1 \%$ & 3 & $3.2 \%$ & & \\
\hline Subjective emptying & Present & 3 & $6.5 \%$ & 3 & $6.1 \%$ & 6 & $6.3 \%$ & FE & 1.000 \\
\hline problems 3 & Absent & 43 & $93.5 \%$ & 46 & $93.9 \%$ & 89 & $93.7 \%$ & & \\
\hline
\end{tabular}

At the third month, the cough stress test (objective cure) showed statistically significant difference between both groups $(89.8 \%$ negative test in group (A) compared to $67.4 \%$ in group $(\mathrm{B})(\mathrm{P}=0.007)$ as shown in table (5). The ICIQ-SF score (subjective cure), however; was statistically insignificant for both groups as shown in table (5).Persistently, the frequency of micturition before and after surgery in both groups represented a statistically significant difference at the third month. Moreover, the percentage of women experienced groin pain in group (B) shrunk to $45.7 \%$, though, the statistically significant difference was still presented as shown in table (5).

\section{The sixth month follow-up:}

At this visit, the proportion of women experienced negative cough stress test (objective cure) continued to rise in both groups. Despite the bounce in group (B) percentage (79.2\% after $67.4 \%$ at the third month) compared to group (A) rise (93.3\% after $89.8 \%$ at the previous visit), yet there was statistically significant difference between both groups $(\mathrm{P}=0.033)$ as shown in table (6). 
Table (6): Comparison between both studied groups as regard post-operative urinary symptoms and pain scores at the $6^{\text {th }}$ month follow up.

\begin{tabular}{|c|c|c|c|c|c|c|c|c|c|}
\hline & \multicolumn{6}{|c|}{ Groups } & \multicolumn{2}{|c|}{ Tests of significance } \\
\hline & & \multicolumn{2}{|c|}{$\begin{array}{c}\text { TOT } \\
(n=48)\end{array}$} & \multicolumn{2}{|c|}{$\begin{array}{c}\text { SIMS } \\
(n=49)\end{array}$} & \multicolumn{2}{|c|}{$\begin{array}{c}\text { Total } \\
(\mathbf{n}=97)\end{array}$} & Test statistic & $\mathbf{p}$ \\
\hline \multirow{2}{*}{$\begin{array}{l}\text { Cough stress } \\
\text { test (objective } \\
\text { cure) } 6\end{array}$} & Positive & 10 & $20.8 \%$ & 3 & $6.1 \%$ & 13 & $13.4 \%$ & \multirow[t]{2}{*}{$\mathrm{X}_{\mathrm{ChS}}^{2}=4.521$} & \multirow[t]{2}{*}{$0.033^{*}$} \\
\hline & Negative & 38 & $79.2 \%$ & 46 & $93.9 \%$ & 84 & $86.6 \%$ & & \\
\hline \multirow{3}{*}{$\begin{array}{l}\text { ICIQ-SF } \\
\text { score } 6\end{array}$} & Min-Max & \multicolumn{2}{|c|}{$0.0-18.0$} & \multicolumn{2}{|c|}{$0.0-16.0$} & \multicolumn{2}{|c|}{$0.0-18.0$} & \multirow[t]{3}{*}{$\mathrm{Z}=1.390$} & \multirow[t]{3}{*}{0.165} \\
\hline & $\begin{array}{l}\text { Median } \\
\text { (IOR) }\end{array}$ & \multicolumn{2}{|c|}{$0.0(0.0-6.0)$} & \multicolumn{2}{|c|}{$0.0(0.0-0.0)$} & \multicolumn{2}{|c|}{$0.0(0.0-6.0)$} & & \\
\hline & Mean rank & \multicolumn{2}{|c|}{50.6} & \multicolumn{2}{|r|}{443} & & & & \\
\hline \multirow{6}{*}{$\begin{array}{l}\text { ICIQ question } \\
\text { 3-6 }\end{array}$} & Never & 34 & $70.8 \%$ & 38 & $77.6 \%$ & 72 & $74.2 \%$ & \multirow[t]{6}{*}{$\mathrm{X}_{\mathrm{FFH}}^{2}=4.876$} & \multirow[t]{6}{*}{0.301} \\
\hline & once/week & 3 & $6.3 \%$ & 6 & $12.2 \%$ & 9 & $9.3 \%$ & & \\
\hline & $\begin{array}{l}2-3 \\
\text { times/week }\end{array}$ & 3 & $6.3 \%$ & 1 & $2.0 \%$ & 4 & $4.1 \%$ & & \\
\hline & Daily & 5 & $10.4 \%$ & 4 & $8.2 \%$ & 9 & $9.3 \%$ & & \\
\hline & Several & 3 & $6.3 \%$ & 0 & $0.0 \%$ & 3 & $3.1 \%$ & & \\
\hline & All time & 0 & $0.0 \%$ & 0 & $0.0 \%$ & 0 & $0.0 \%$ & & \\
\hline \multirow{2}{*}{$\begin{array}{l}\text { Number of } \\
\text { Micturitions } \\
\text { per day } 6\end{array}$} & Min- Max & \multicolumn{2}{|c|}{$6.0-8.0$} & \multicolumn{2}{|c|}{$5.0-8.0$} & \multicolumn{2}{|c|}{$5.0-8.0$} & \multirow[t]{2}{*}{$\mathrm{t}=0.539$} & \multirow[t]{2}{*}{0.591} \\
\hline & Mean \pm SD & \multicolumn{2}{|c|}{$6.9 \pm 0.8$} & \multicolumn{2}{|c|}{$7.0 \pm 1.1$} & \multicolumn{2}{|c|}{$6.9 \pm 1.0$} & & \\
\hline \multirow{3}{*}{$\begin{array}{l}\text { Incontinence } \\
\text { episodes per } \\
\text { day } 6\end{array}$} & Min-Max & & $0-4.0$ & & $0-4.0$ & & $0-4.0$ & $\mathrm{Z}=2.082$ & $0.037 *$ \\
\hline & $\begin{array}{l}\text { Median } \\
\text { (IQR) }\end{array}$ & 0.0 & $(0.0-2.0)$ & 0.0 & $(0.0-0.0)$ & 0.0 & $(0.0-1.0)$ & & \\
\hline & Mean rank & & 53.7 & & 44.4 & & & & \\
\hline Groin Pain 6 & Present & 19 & $39.6 \%$ & 0 & $0.0 \%$ & 19 & $19.6 \%$ & $\mathrm{X}_{\mathrm{ChS}}^{2}=24.120$ & $<0.001 *$ \\
\hline & Absent & 29 & $60.4 \%$ & 49 & $100.0 \%$ & 78 & $80.4 \%$ & & \\
\hline Post Voiding & Less 100 & 48 & $100.0 \%$ & 49 & $100.0 \%$ & 97 & $100.0 \%$ & NA & NA \\
\hline residual 6 & More 100 & 0 & $0.0 \%$ & 0 & $0.0 \%$ & 0 & $0.0 \%$ & & \\
\hline Subjective & Present & 3 & $6.3 \%$ & 3 & $6.1 \%$ & 6 & $6.2 \%$ & FE & 1.000 \\
\hline $\begin{array}{l}\text { emptying } \\
\text { problems } 6\end{array}$ & Absent & 45 & $93.8 \%$ & 46 & $93.9 \%$ & 91 & $93.8 \%$ & & \\
\hline
\end{tabular}

Simultaneously, the ICIQ-SF score (subjective cure) showed lower results than the previous visit for both groups, yet, without significant difference in both groups $(\mathrm{P}=0.165)$.

Additionally, a statistically significant difference occurred between the two groups regarding the incontinence episodes per day with an inter quartile range (IQR) of (0.0-0.0) for group (A) and (0.0-2.0) for group (B) $(\mathrm{P}=0.037)$ as shown in table (6).

Regarding the groin pain, the percentage of women experienced such pain continued to drop in group (B) with time and the statistically significant difference between the two groups was still presented $(\mathrm{P}<0.001)$ as shown in table $(6)$.

\section{$>$ The twelfth month follow-up:}

Regarding the cough stress test after one year follow up, the number of participants who reported negative results showed continuous progress in group (B) (42 women (89\%) after 38 $(79.2 \%)$ in $6^{\text {th }}$ month visit). On the other hand, group (A) women showed a little drop (44 women (91.7\%) after 46 (93.9) in $6^{\text {th }}$ month visit). Yet, the two groups showed insignificant difference at this follow up stage $(\mathrm{P}=0.74)$ as shown in table (7). 
Table (7): Comparison between both studied groups as regard post-operative urinary symptoms and pain scores at the $12^{\text {th }}$ month follow up.

\begin{tabular}{|c|c|c|c|c|c|c|c|c|c|}
\hline & \multicolumn{6}{|c|}{ Groups } & \multicolumn{2}{|c|}{ Tests of significance } \\
\hline & & \multicolumn{2}{|c|}{$\begin{array}{c}\text { TOT } \\
(\mathrm{n}=47)\end{array}$} & \multicolumn{2}{|c|}{$\begin{array}{c}\text { SIMS } \\
(n=48)\end{array}$} & \multicolumn{2}{|c|}{$\begin{array}{c}\text { Total } \\
(n=95)\end{array}$} & Test statistic & $\mathbf{p}$ \\
\hline \multirow{2}{*}{$\begin{array}{l}\text { Cough stress } \\
\text { (objective cure) } 12\end{array}$} & Positive & 5 & $10.6 \%$ & 4 & $8.3 \%$ & 9 & $9.5 \%$ & \multirow[t]{2}{*}{ FE } & \multirow[t]{2}{*}{0.740} \\
\hline & Negative & 42 & $89.4 \%$ & 44 & $91.7 \%$ & 86 & $90.5 \%$ & & \\
\hline \multirow[t]{3}{*}{ ICIQ-SF score 12} & Min-Max & \multicolumn{2}{|c|}{$0.0-18.0$} & \multicolumn{2}{|c|}{$0.0-16.0$} & \multicolumn{2}{|c|}{$0.0-18.0$} & \multirow[t]{3}{*}{$\mathrm{Z}=2.351$} & \multirow[t]{3}{*}{$0.019 *$} \\
\hline & $\begin{array}{l}\text { Median } \\
\text { (IQR) }\end{array}$ & \multicolumn{2}{|c|}{$0.0(0.0-5.0)$} & \multicolumn{2}{|c|}{$0.0(0.0-0.0)$} & \multicolumn{2}{|c|}{$0.0(0.0-4.0)$} & & \\
\hline & Mean rank & \multicolumn{2}{|c|}{53.1} & \multicolumn{2}{|r|}{43.0} & & & & \\
\hline \multirow[t]{6}{*}{ ICIQ question 3-12 } & Never & 30 & $63.8 \%$ & 41 & $85.4 \%$ & 71 & $74.7 \%$ & \multirow[t]{6}{*}{$\mathrm{X}_{\mathrm{FFH}}^{2}=11.877$} & \multirow[t]{6}{*}{$0.009 *$} \\
\hline & once/week & 4 & $8.5 \%$ & 3 & $6.3 \%$ & 7 & $7.4 \%$ & & \\
\hline & $\begin{array}{l}2-3 \\
\text { times/week }\end{array}$ & 5 & $10.6 \%$ & 3 & $6.3 \%$ & 8 & $8.4 \%$ & & \\
\hline & Daily & 8 & $17.0 \%$ & 0 & $0.0 \%$ & 8 & $8.4 \%$ & & \\
\hline & Several & 0 & $0.0 \%$ & 1 & $2.1 \%$ & 1 & $1.1 \%$ & & \\
\hline & All time & 0 & $0.0 \%$ & 0 & $0.0 \%$ & 0 & $0.0 \%$ & & \\
\hline \multirow{2}{*}{$\begin{array}{l}\text { Number of micturitions } \\
\text { per day } 12\end{array}$} & Min- Max & \multirow{2}{*}{\multicolumn{2}{|c|}{$\begin{array}{l}6.0-9.0 \\
7.3+0.7\end{array}$}} & \multicolumn{2}{|c|}{$5.0-8.0$} & \multicolumn{2}{|c|}{$5.0-9.0$} & \multirow[t]{2}{*}{$\mathrm{t}=0.860$} & \multirow[t]{2}{*}{0.392} \\
\hline & Mean \pm SD & & & & & & $2 \pm 0.9$ & & \\
\hline \multirow{3}{*}{$\begin{array}{l}\text { Incontinence episodes } \\
\text { per day } 12\end{array}$} & Min-Max & \multicolumn{2}{|c|}{$0.0-4.0$} & \multicolumn{2}{|c|}{$0.0-4.0$} & & $0-4.0$ & $\mathrm{Z}=2.440$ & $0.015 *$ \\
\hline & $\begin{array}{l}\text { Median } \\
\text { (IQR) }\end{array}$ & 0.0 & $(0.0-1.0)$ & 0.0 & $(0.0-0.0)$ & 0.0 & $(0.0-1.0)$ & & \\
\hline & Mean rank & & 53.3 & & 42.8 & & & & \\
\hline Groin Pain 12 & Present & 12 & $25.5 \%$ & 0 & $0.0 \%$ & 12 & $12.6 \%$ & $\mathrm{X}_{\mathrm{ChS}}^{2}=14.027$ & $<0.001^{*}$ \\
\hline & Absent & 35 & $74.5 \%$ & 48 & $100.0 \%$ & 83 & $87.4 \%$ & & \\
\hline Post Voiding residual & Less 100 & 47 & $100.0 \%$ & 48 & $100.0 \%$ & 95 & $100.0 \%$ & NA & NA \\
\hline & More 100 & 0 & $0.0 \%$ & 0 & $0.0 \%$ & 0 & $0.0 \%$ & & \\
\hline Subjective emptying & Present & 3 & $6.4 \%$ & 0 & $0.0 \%$ & 3 & $3.2 \%$ & FE & 0.117 \\
\hline problems 12 & Absent & 44 & $93.6 \%$ & 48 & $100.0 \%$ & 92 & $96.8 \%$ & & \\
\hline
\end{tabular}

Contrarily, there were significant difference between the two groups regarding; ICIQ-SF score, the episodes of incontinence and the groin pain $(\mathrm{P}=0.019,0.015$ and $<0.001$, respectively) as shown in table (7).

\section{DISCUSSION}

After fulfilling the inclusion criteria, there was no significant difference between both groups (SIMS as group (A) and TOT as group(B)) regarding the mean age of the participants ( $46.3 \pm 10.5$ years) which was in agreement with Rudnicki et al. (6) and Basu and Duckett ${ }^{(7)}$. In parallel, a slight increase in mean age was found in a study by Chang $\boldsymbol{e t} \mathbf{a l}{ }^{\left({ }^{(8)}\right.}$ with a mean age of 59.9 years. There was significant association of increasing age and presence of urinary incontinence in women ${ }^{(9)}$.

Additionally, there were no statistically significant differences between the two groups regarding the other personal descriptive parameters such as the women's weight, height, body mass index, parity and menopausal status. This was also the case in Rudnicki et al. ${ }^{(6)}$, Neuman et al. ${ }^{(10)}$ and Chang et al. (8) studies.

Regarding the women main complaint, the principle symptom in both groups was leakage of urine with cough or sneezing (97\%) and only 3 women in group (B) reported both urgency and involuntary leakage. This was statistically insignificant between both

groups. In the contrary, the mixed urinary incontinence represented up to $23.9 \%$ in the study Rudnicki et $\boldsymbol{a l} .{ }^{(6)}$ and raised up to $58 \%$ in Bianchi-Ferraro et al. ${ }^{(11)}$ study. In our study, we preferred to prescribe medical treatment -initially- for women complaining of mixed urinary incontinence in order to reduce the bias and the heterogeneity of results, especially, the ICIQ-SF scores. Specially, this trend was recommended by NICE guidelines ${ }^{(\mathbf{1 2})}$.

The local examination of the studied women showed non-significant difference between both groups. There was an agreement to exclude women needing extensive concomitant surgery in order not to add bias in the results of the study. The duration of the studied procedure was calculated separately from other concomitant surgery if indicated.

Baseline characteristics of bladder function 
As it was the main inclusion criterion in our study, all women presented with positive cough stress test, and this was in accordance with Kocjancic et al. (13).

Conversely, women included in our study reported a mean of $5.2 \pm 0.4$ to $5.4 \pm 0.8$ visits to the bathroom for micturition (group (A) and (B), respectively). These figures were quite higher in other studies, where number of micturition's per day reached a mean of 7.2 \pm 2.2 Rudnicki et al. ${ }^{(6)}$. This difference may be attributed to increased percentage of women complaining mixed incontinence in Rudnicki's trial (23.9\% rather than $16 \%$ in our study) which necessitate more frequent bathroom visits due to urgency.

\section{Operative data}

In our study, a statistically significant difference was found between both groups regarding the duration of the procedure. While it ranged between 12 to 17 minutes in SIMS group with a mean of $14.1 \pm$ 1.4 minutes, it reached 15 to 22 minutes with a mean of $17.7 \pm 2.0$ in TOT group. The SIMS was superior to TOT in terms of shorter operative time in many trials Chang et al. ${ }^{(8)}$ and Rudnicki et al. ${ }^{(6)}$.

In the contrary, in a study by Neuman et al. ${ }^{(10)}$, there was no difference between the groups regarding the duration of the procedure.

This study aimed to compare the safety between the two tapes SIMS and TOT. Though, we failed to find a significant difference between the two groups regarding surgical complications. This finding was in agreement with Chang et al. ${ }^{\left({ }^{(8)}\right.}$, Neuman et al. (10) and Rudnicki et al. (6). Unsurprisingly, the encountered complications in most of studies including the current study- were mostly in the form of bladder injury in case of TOT and localized hematomas in case of SIMS ${ }^{(14)}$. Consequently, the SIMS were produced to reduce such morbidity ${ }^{\left({ }^{15}\right)}$.

\section{Post-operative follow-up:}

Regarding post-operative groin pain, the current study showed a statistically significant difference in favor of SIMS with VAS scores around $5 / 10$. Other studies proved the same results regarding both vaginal and thigh pain, with scores higher than $5 / 10$ in TOT rather than SIMS ${ }^{(16)}$. Moreover, even in low scores (VAS scores 3/10), the pain scores were still lower with SIMS than with the TOT procedure ${ }^{(\mathbf{1 0})}$.

Conversely, no significant difference in VAS scores between the two procedures was found after each postoperative follow-up in other trials ${ }^{(8)}$.

Regarding other one-week post-operative parameters, there were no statistically significant differences between both groups regarding; cough stress test, post-voiding residual urine, excessive vaginal discharge, subjective emptying problems, urge incontinence and wound infection. These findings came in accordance with other studies by Neuman et al. ${ }^{(10)}$, Rudnicki et al. ${ }^{(6)}$ and the systematic review and metaanalysis by Mostafa et al. ${ }^{(16)}$.

\section{Follow-up visits:}

\section{$>$ The first month follow-up visit:}

Most of the studies scheduled the follow up visits at $3^{\text {rd }}, 6^{\text {th }}$ and $12^{\text {th }}$ months. Contrarily, the first month follow-up was added in this study. There was a statistically significant difference between the frequency of micturition before and after surgery in both groups and this trend was resulted from the advice of increasing the frequency of micturition as a sort of bladder training. Additionally, the percentage of women experienced groin pain in group (B) outnumbered their counterparts in group (A).

\section{$>$ The third month follow-up visit:}

Regarding the objective and subjective cure rates at the third month, the cough stress test (objective cure) showed statistically significant difference between both groups despite that the ICIQ-SF score (subjective cure) failed to achieve the same result. These differences were quite different from those of Rudnicki et al. ${ }^{(6)}$ which showed no difference between the groups.

Regarding the safety (complication rates), the percentage of women experienced groin pain in group (B) continued to decrease, though, the statistically significant difference was still presented.

\section{$>$ The sixth month follow-up visit:}

The objective cure rate continued to rise in both groups. Yet, there was statistically significant difference between both groups $(93.3 \%$ in SIMS compared to $79.2 \%$ in TOT). Conversely, these results were totally different from those in both Chang $\boldsymbol{e t ~ a l . ~}{ }^{(8)}$ and Basu and Duckett ${ }^{(7)}$ studied which showed better results in favor of non-SIMS tapes. However, in another non-comparative studies, the cough stress test was negative for up to $(92.2 \%)$ of cases at 6 months using the same SIMS as the current study Kocjancic et al. ${ }^{(13)}$.

\section{$>$ The twelfth month follow-up visit:}

Regarding the cough stress test (objective cure), no significant difference was found between the two groups after one-year follow-up. The TOT group showed continuous progress to achieve $89 \%$ against $91.7 \%$ in SIMS group. These statistics were in agreement with Rudnicki et al. ${ }^{(6)}$ who found equal objective cure rate in both groups.

Consequently, regarding the objective cure measure, there were insignificant differences between the two groups in the absolute risk reduction (ARR) $(2.3 \%$, CI: $0-15.28 \%, \mathrm{p}=0.555)$, relative risk (RR) $(0.78$, CI: $0.22-2.74, p=0.702)$ and relative risk reduction $($ RRR) $(0.22, \mathrm{CI}: 0-0.78, \mathrm{p}=0.271)$. This calculation was in agreement with Rudnicki et al. ${ }^{\left({ }^{(6)} \text {, }\right.}$ where relative risks (RR) calculation indicated no difference between the two groups regarding objective [RR 0.938, 95\% confidence interval (CI) 0.705-1.249, $\mathrm{p}=0.691$; no leakage vs. leakage $]$ and subjective cure rate (RR 0.88; 95\% CI 0.696- 1.112, p = 0.627; never leakage vs. any leakage). 
However, regarding the subjective cure measure (ICIQ-SF score), a significant difference was found between both groups in ARR $(21.6 \%$, CI: 4.1 $37.7 \%, \mathrm{p}=0.012)$, RR $(0.4, \mathrm{CI}: 0.2-0.9, \mathrm{p}=0.023)$ and RRR (0.60, CI: $0.12-0.82, \mathrm{p}=0.001)$.

This unexplained difference between both the subjective and objective cure rates in each group might be attributed to the definition of cure and the individual expectations between the research team and the candidates as reported by Hilton and Robinson ${ }^{(17)}$. Moreover, in accordance with Chang et al. ${ }^{(8)}$, women in the SIMS group had less frequency of intent to treat than those in the TOT group. Whereas, this figure was 43 based upon objective cure, it dropped to only 5 women needed to be treated based upon subjective cure measure.

After one year follow up, the post-operative pain profile was significantly lower in the SIMS group than the TOT group. This is because that the standard TOT requires three incisions (one vaginal, two in the groin) and the passage of trocars through adductor muscles which is not presented in SIMS ${ }^{(15)}$. This difference in pain perception was in agreement with most of previous studies Mostafa et al. ${ }^{(16)}$, Chang et $a{ }^{\left({ }^{(8)}\right.}$ and Rudnicki et al. ${ }^{\left({ }^{(6)} \text {. }\right.}$

Regarding other far complications, no reports were found of mesh erosion, or foreign body sensation through 12 months. These data were in agreement with Kocjancic et al. ${ }^{(13)}$.

\section{CONCLUSION}

The 1-year follow-up results of this prospective trial indicate that both procedures appear to be equally effective for the treatment of SUI as regard the objective cure rates, whereas the SIMS procedure showed higher subjective cure rates than the TOT procedure. Regarding pain perception, the SIMS showed less postoperative groin pain compared to the TOT.

Moreover, the operative time was shorter in the SIMS compared to the TOT. Both procedures were associated with a similar incidence of perioperative and postoperative complications. Overall complication rates were also similar, with the exception of groin pain.

\section{REFERENCES}

1. Capobianco G, Madonia M, Morelli $S$ et al. (2018): Management of female stress urinary incontinence: A care pathway and update. Maturitas, 109:32-38.

2. Mostafa A, Agur W, Abdel-All M et al. (2013): Multicenter prospective randomized study of singleincision mini-sling vs tension-free vaginal tape-obturator in management of female stress urinary incontinence: A minimum of 1-year follow-up. Urology, 82(3): 552-559.

3. Novara G, Ficarra V, Boscolo-Berto $\mathrm{R}$ et al. (2007): Tension-free midurethral slings in the treatment of female stress urinary incontinence: A systematic review and meta- analysis of randomized controlled trials of effectiveness. European Urology, 52(3): 663-679.

4. Nambiar A, Cody JD, Jeffery ST et al. (2017): Singleincision sling operations for urinary incontinence in women. doi/ CD008709.pub3/epdf/full $10.1002 / 14651858$.

5. Swift SE, Yoon EA (1999): Test-retest reliability of the cough stress test in the evaluation of urinary incontinence. Obstetrics \& Gynecology, 94: 99-102.

6. Rudnicki M, Von Bothmer- Ostling K, Holstad A et al. (2017): Adjustable mini- sling compared with conventional mid- urethral slings in women with urinary incontinence. A randomized controlled trial. Acta Obstetricia et Gynecologica Scandinavica, 96(11): 13471356.

7. Basu M, Duckett J (2010): A randomised trial of a retropubic tension- free vaginal tape versus a mini- sling for stress incontinence. BJOG: An International Journal of Obstetrics \& Gynaecology, 117(6): 730-735.

8. Chang CP, Chang WH, Hsu YM et al. (2015): Comparison of single-incision mini-slings (ajust) and standard transobturator midurethral slings (align) in the management of female stress urinary incontinence: A 1year follow-up. Taiwanese Journal of Obstetrics and Gynecology, 54(6): 726-730.

9. Agarwal BK, Agarwal N (2017): Urinary incontinence: Prevalence, risk factors, impact on quality of life and treatment seeking behaviour among middle aged women. International Surgery Journal, 4(6): 1953-1958.

10. Neuman $M$, Sosnovski V, Kais $M$ et al. (2011): Transobturator vs single-incision suburethral mini-slings for treatment of female stress urinary incontinence: Early postoperative pain and 3-year follow-up. Journal of Minimally Invasive Gynecology, 18(6): 769-773.

11. Bianchi-Ferraro AMH, Jarmy-Di Bella ZI, Castro RDA et al. (2013): Single-incision sling compared with transobturator sling for treating stress urinary incontinence: A randomized controlled trial. International Urogynecology Journal, 24(9): 1459-1465.

12. National GAU (2019): Urinary incontinence and pelvic organ prolapse in women: Management. https://www.nice.org.uk/guidance/ng123

13. Kocjancic E, Tu LM, Erickson T et al. (2014): The safety and efficacy of a new adjustable single incision sling for female stress urinary incontinence. The Journal of Urology, 192(5): 1477-1482.

14. Minaglia S, Özel B, Klutke C et al. (2004): Bladder injury during transobturator sling. Urology, 64(2): 376-377.

15. Morán E, Pérez- Ardavín J, Sánchez JV et al. (2019): Mid- term safety and efficacy of the altis ${ }^{\circledR}$ singleincision sling for female stress urinary incontinence: Less mesh, same results. BJU International, 123(5A): E51-E56.

16. Mostafa A, Lim CP, Hopper L et al. (2014): Singleincision mini-slings versus standard midurethral slings in surgical management of female stress urinary incontinence: An updated systematic review and metaanalysis of effectiveness and complications. European Urology, 65(2): 402-427.

17. Hilton P, Robinson $D$ (2011): Defining cure. Neurourology and Urodynamics, 30(5): 741-745. 